\title{
Competências essenciais de promoção da saúde na formação do enfermeiro: revisão integrativa
}

Essential health promotion competencies in nursing training: an integrative review Competencias esenciales de promoción de la salud en la formación del enfermero: revisión integradora

Priscila de Oliveira Carvalho ${ }^{1,2}$ id https://orcid.org/0000-0002-2374-0592

Luciane Sá de Andrade² id https://orcid.org/0000-0002-8703-7919

Wanderlei Abadio de Oliveira ${ }^{3}$ io https://orcid.org/0000-0002-3146-8197,

Lívia Masson² id https://orcid.org/0000-0003-0987-7901

Jorge Luiz da Silva ${ }^{4}$ io https://orcid.org/0000-0002-3727-8490

Marta Angélica lossi Silva² io https://orcid.org/0000-0002-9967-8158

\section{Como citar:}

Carvalho PO, Andrade LS, Oliveira WA, Masson L, Silva JL, Silva MA. Competências essenciais de promoção da saúde na formação do enfermeiro: revisão integrativa. Acta Paul Enferm. 2021;34:eAPE02753.

DOI

http://dx.doi.org/10.37689/actaape/2021AR02753

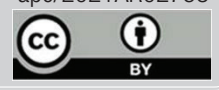

Descritores

Competência profissional; Promoção da Saúde; Capacitacão profissional; Enfermeiros

Keywords

Professional competence; Health promotion; Professional training; Nurses

Descriptores

Competencia profissional; Promoción de la salud; Capacitación profesional; Enfermeros

\section{Submetido \\ 29 de Setembro de 2019 \\ Aceito \\ 8 de Setembro de 2020}

Autor correspondente

Priscila de Oliveira Carvalho

E-mail: priscilaoc@ifsp.edu.br

\section{Resumo}

Objetivo: Analisar evidências disponíveis na literatura sobre as competências essenciais de promoção da saúde relacionadas ao Projeto, "Desenvolvendo competências e padrões profissionais para a construção da capacidade em promoção da saúde na Europa" (CompHP), na formação do enfermeiro no contexto brasileiro.

Métodos: Revisão integrativa de literatura nas bases de dados PUBMED, Web of Science, CINAHL, LILACS, BDENF e Biblioteca Eletrônica Scielo, com descritores "Enfermagem", "Promoção da Saúde" e palavras-chave "Competências" e "CompHP". Foram elegíveis artigos publicados a partir de 2011, nos idiomas português, inglês e espanhol. A questão norteadora e as buscas nas bases de dados ocorreram a partir da Estratégia PVO.

Resultados: Seis publicações foram incluídas, sendo estabelecidas duas categorias temáticas: Desenvolvimento de Competências do CompHP na formação do enfermeiro; Ensino das Competências de Promoção da Saúde. As competências do CompHP têm sido desenvolvidas durante a formação do enfermeiro, contudo, apenas três delas com regularidade: Possibilidade de mudança; Parceria e Liderança.

Conclusão: Este estudo amplia a compreensão sobre como alguns processos relacionados ao CompHP têm sido incorporados à formação de enfermeiros, além de versar sobre sua importância para a formação crítica e reflexiva a partir da incorporação das competências essenciais no currículo de cursos de graduação de enfermagem no cenário brasileiro.

\section{Abstract}

Objective: to analyze the evidence available in the literature on essential health promotion competencies related to Developing Competencies and Professional Standards for Health Promotion Capacity Building in Europe (CompHP) project, in nursing training in the Brazilian context.

Methods: this is an, integrative literature review in the PUBMED, Web of Science, CINAHL, LILACS, BDENF and Scielo Electronic Library databases, with descriptors "Nursing", "Health Promotion" and keywords "Competencies" and "CompHP". Articles published since 2011, in Portuguese, English and Spanish were eligible. The guiding question and the searches in the databases occurred from PVO strategy.

Results: six publications were included, with two thematic categories being established: Development of CompHP Competencies in nursing training; Teaching Health Promotion Competencies. CompHP competencies have been developed during nursing training; however, only three of them regularly: enable change, mediation through partnership and leadership. 
Conclusion: this study expands the understanding of how some processes related to CompHP have been incorporated into nursing training, in addition to addressing their importance for critical and reflective training from the incorporation of essential competencies in the curriculum of undergraduate nursing courses in the Brazilian setting.

\section{Resumen}

Objetivo: Analizar las evidencias disponibles en la literatura sobre las competencias esenciales de promoción de la salud relacionadas con el proyecto "Desarrollo de competencias y criterios profesionales en la construcción de capacidades para la promoción de la salud en Europa" (CompHP) en la formación del enfermero en el contexto brasileño.

Métodos: Revisión integradora de literatura en las bases de datos PUBMED, Web of Science, CINAHL, LILACS, BDENF y Biblioteca Electrónica Scielo, con descriptores "Enfermería", "Promoción de la salud" y palabras clave "Competencias" y "CompHP". Los artículos elegibles fueron los publicados a partir de 2011, en portugués, inglés y español. La pregunta orientadora y las búsquedas en las bases de datos se realizaron siguiendo la estrategia PVO.

Resultados: Se incluyeron seis publicaciones y se establecieron dos categorías temáticas: Desarrollo de competencias del CompHP en la formación del enfermero; Enseñanza de las competencias de promoción de la salud. Las competencias del CompHP fueron desarrolladas durante la formación del enfermero, sin embargo, solo tres de ellas con regularidad: Posibilidad de cambio, Colaboración y Liderazgo.

Conclusión: Este estudio amplía la comprensión sobre cómo algunos procesos relacionados con el CompHP fueron incorporados a la formación de enfermeros, además de abordar su importancia para la formación crítica y reflexiva a partir de la incorporación de las competencias esenciales en el diseño curricular de carreras de grado de enfermería en el escenario brasileño.

\section{Introdução}

A Promoção da Saúde (PS) é entendida como um processo de capacitação dos sujeitos para o cuidado em saúde, buscando a autonomia no âmbito individual e coletivo, a fim de melhorar os determinantes do processo saúde-doença e, consequentemente, a qualidade de vida. Emerge no mundo na década de 1970 , por meio do relatório de Lalonde, a partir de um entendimento de que o modelo de atençáo, focado no tratamento de doenças e na reabilitação, não correspondia ao conceito ampliado de saúde. ${ }^{(1,2)}$

Desde esse período, sucessivas conferências promovidas pela Organização Mundial de Saúde (OMS) têm ocorrido para debater e pactuar políticas públicas que atendam uma nova lógica de agir em saúde e de formação em saúde, em seus diferentes campos, ${ }^{(2)}$ uma vez que, sem o compromisso político em ações e investimentos para tratar de saúde e equidade, sem parceria e reivindicação entre os diversos atores sociais, o setor de saúde não atingirá os níveis de saúde almejados para a população. ${ }^{(2)}$

No Brasil, avanços foram alcançados quando a Política Nacional de Promoção da Saúde - PNPS iniciou a formulação, implementação e concretização de açóes que puderam (re)construir um modelo de atenção à saúde que priorizou a melhoria da qualidade de vida dos sujeitos e do coletivo, por meio da ampliação e qualificação das ações de PS nos serviços e na gestão do Sistema Único de Saúde. ${ }^{(3)}$
No campo da saúde há uma preocupação com a formação profissional específica em PS. Neste sentido, diversas iniciativas, sobretudo internacionais, têm contribuído para a formação de recursos humanos em saúde, com a construção e utilização de competências para a PS. Países como Canadá, Austrália e outros do continente Europeu têm se destacado nessa direção. Contudo, na América Latina é preciso avançar mais, considerando a inclusão de conhecimentos e habilidades sobre estratégias de promoção da saúde na formação dos profissionais da saúde. ${ }^{(4)}$

Em 2009, a União Internacional de Promoção e Educação para a Saúde iniciou a idealização do manual a partir do Projeto "Desenvolvendo competências e padróes profissionais para a construção da capacidade em promoção da saúde na Europa (CompHP)". O projeto contribuiu para elevar o núcleo de competências do Galway com padróes profissionais e propôs um quadro de acreditação para a prática de PS, com objetivo de impactar positivamente a formação e os recursos humanos do continente europeu. ${ }^{(4,5)}$

As competências essenciais do projeto CompHP são descritas como "um conjunto de papéis esperados aos profissionais de saúde, para que sejam capazes de trabalhar de forma eficiente". Apesar de ter sido desenvolvido para uma realidade específica, o CompHP foi construído a partir de uma ampla revisão de literatura de competências essenciais de PS existentes, e diversas rodadas de consultas a inúme- 
ros especialistas, a fim de impactar uma realidade multicultural. ${ }^{(5-7)}$

As competências essenciais para atuar na PS definidas pelo projeto incluem um conjunto de 68 declaraçóes de competências, dispostos em onze domínios, a saber: Possibilidade de Mudanças, Advocacia em Saúde, Mediação por meio de Parceiros, Comunicação, Liderança, Diagnóstico, Planejamento, Implementação, Avaliação e Pesquisa. Por sua vez, essas competências devem ser desenvolvidas e orientadas pelos domínios Valores Éticos e Conhecimentos em PS. ${ }^{(5-8)}$

No contexto da enfermagem, ressalta-se que o modelo tradicional de ensino, mesmo com os avanços na organização curricular, não tem sido capaz de capacitar o enfermeiro generalista para atuar efetivamente na PS, um dos eixos estruturantes do Sistema Único de Saúde, no caso brasileiro. Analisar o ensino das competências para a PS na formação do enfermeiro, identificando experimentaçóes e experiências nesse processo ${ }^{(9)}$ Ademais, a inserção da promoção da saúde nos currículos, em geral, tem sido relacionada às atividades de educação em saúde em diferentes contextos. ${ }^{(9)}$

Por trás desse cenário há um embate das diversas perspectivas de PS, refletindo a necessária e ainda a baixa solidez da intersetorialidade na elaboração da PNPS, que menciona a importância de realizar parcerias, mas, em sua formulação inicial, a pactuação e diálogo com os diversos setores da sociedade procederam de forma insuficiente. ${ }^{(3,10)}$

Por outro lado, há um consenso estabelecido na literatura sobre a importância do desenvolvimento de uma rede efetiva e sustentável de profissionais envolvidos com as competências para PS. ${ }^{(4,5,9)}$ Assim, este estudo objetivou analisar a produção do conhecimento científico acerca de competências de PS relacionadas ao CompHP e pertinentes à formação do enfermeiro no Brasil.

\section{Métodos}

A revisão integrativa foi desenvolvida seguindo-se as etapas: elaboração da questão de pesquisa; estabelecimento dos critérios de inclusão e exclusão; busca de estudos primários; avaliação dos estudos incluídos; categorização dos estudos; interpretação dos resultados e síntese dos resultados evidenciados. ${ }^{(11)}$

Utilizou-se a estratégia PVO (Population or Problem, Variables e Outcomes) e chegou-se à questão norteadora: Quais são as evidências presentes na literatura científica acerca do desenvolvimento de competências de PS relacionadas ao CompHP na formação do enfermeiro? Onde o P seria o enfermeiro, V a formação para Promoção da Saúde e O a identificação de aspectos de competência de PS relacionada ao projeto CompHP. ${ }^{(12)}$

Para a seleção dos artigos, realizou-se buscas em setembro de 2018 nas bases de dados: Cumulative Index to Nursing and Allied Health Literature (CINAHL), NationalLibrary of Medicine (PubMed), Web of Science (WOS), Literatura Latino-americana e do Caribe em Ciências da Saúde (LILACS), Banco de Dados de Enfermagem (BDENF) e Scientific Eletronic Library Online (SciELO). Para escolha de descritores e palavras-chave, foi realizado o levantamento de termos a partir da Estratégia PVO, e efetuado uma consulta ao vocabulário próprio das bases envolvidas no estudo. Após análise de combinaçóes de descritores controlados, optou-se pelo uso dos descritores nursing, health promotion e as palavras-chaves competence, CompHP, a fim de expandir os resultados para artigos publicados e que possivelmente não tivessem sofrido o processo de análise para indexação dos termos. Fizemos o uso de operadores boleanos $A N D$ e $O R$ e de truncamentos $\left(\$ \mathrm{e}^{*}\right)$. Os termos dos descritores foram adotados de forma trilíngue, português inglês e espanhol, nas bases nacionais, e utilizamos o cruzamento (Competence OR Competencies OR CompHP) AND bealth promotion" AND (nursing OR Nurse*).

A busca e a seleção de artigos foram realizadas por duas pesquisadoras de forma independente. Os critérios de inclusão foram: artigos de estudos primários publicados nos idiomas português, inglês e espanhol, que abordassem a temática do desenvolvimento de competências em promoção da saúde analisados à luz do referencial CompHP; periódicos indexados nas bases de dados selecionadas, entre o período de 2011 a julho de 2018, ou seja, desde a publicação do $1^{\circ}$ manual do CompHP - "The 
Core Competencies Framework for Health Promotion Handebook", publicado em 2011. ${ }^{(8)}$ Os critérios de exclusão foram: revisóes, teses, monografias, dissertações, editoriais, respostas ao editor, cartas e relatos de experiência. Em casos de discordância entre as pesquisadoras foi realizado consenso para seleção.

Foram realizados filtros prévios, no tangente ao período e idiomas nas bases de dados escolhidas. A partir dos resultados das estratégias de busca nas bases, geramos um arquivo que foi importado para o gerenciador de referências EndNote e criadas pastas para cada base. Posteriormente, excluíram-se estudos duplicados, que não abordavam a temática após leitura de título e resumo, e provenientes de documentos incompatíveis para inclusão. Foram selecionados 17 artigos para leitura na íntegra e seis incluídos nesta revisão. A seleção dos estudos seguiu as recomendaçôes do método Preferred Reporting Items for Systematic Review sand Meta-Analyses- PRISMA com adaptação do fluxograma (Figura 1). ${ }^{(13)}$

Para extração das informaçóes e análise dos artigos selecionados foi criada uma matriz-síntese contemplando itens como identificação do estudo, idioma, país de origem, desenho do estudo, tamanho da amostra, método de análise, resultados encontrados, conclusóes/recomendações. Após extração dos dados procedeu-se uma análise de dados dos fragmentos de resultados/ conclusôes de cada estudo, com leitura e releitura dos dados extraídos, reunião de dados e categorização de tema e produção de um relatório final, articulando o objetivo do estudo e os dados encontrados. A classificação do nível de evidência dos estudos seguiu: Nível I, provenientes de revisão sistemática ou metanálise; o Nível II, ensaio clínico controlado randomizado; Nível III, ensaio clínico controlado sem randomização; Nível IV, estudos de coorte ou de caso-controle; Nível V, originários de revisão sistemática de estudos descritivos ou qualitativos; Nível VI, estudo descritivo ou qualitativo; nível VII, provenientes de opinião de autoridades e / ou relatórios de comitê de especialistas. ${ }^{(14)}$

\section{Resultados}

Os seis artigos incluídos nesta revisão foram desenvolvidos no Brasil e publicados nos últimos quatro anos. Quanto aos pesquisadores, cinco estudos tinham como autores principais enfermeiros e um produzido por fisioterapeutas. Houve um predomínio de instituiçóes na região Sudeste e no estado de Minas Gerais, número maior de instituições públicas que privadas, mas identificou-se quatro estudos

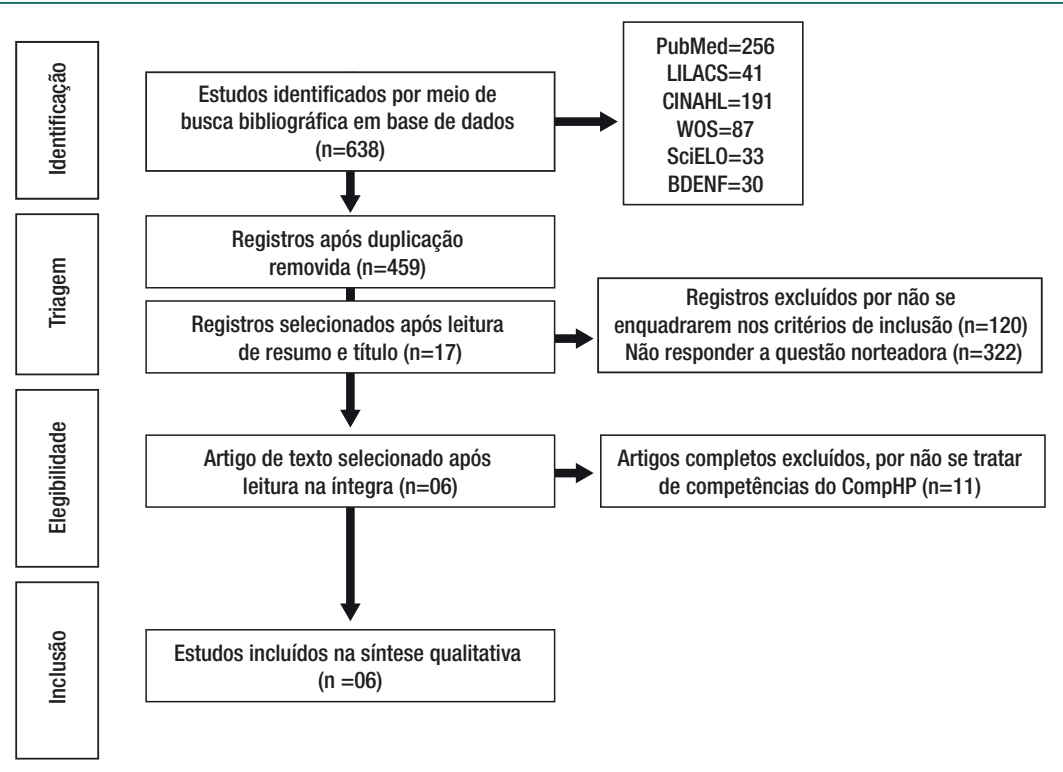

Fonte: Shamseer L, Moher D, Clarke M, Ghersi D, Liberati A, Petticrew M, et al.; PRISMA-P Group. Preferred reporting items for systematic review and meta-analysis protocols (PRISMA-P) 2015: elaboration and explanation. BMJ. 2015;350 jan02 1:g7647.(13)

Figura1. Fluxograma da busca dos estudos primários nas bases de dados selecionadas ${ }^{(13)}$ 
realizados no Nordeste, um na região sul e um na regiâo centro-oeste. A caracterização e síntese dos principais achados nos artigos encontrados estáo apresentadas no quadro 1 .

O quantitativo de estudos selecionados, tendo em vista a rigorosidade metodológica empregada, denota que as discussóes sobre as Competências para Promoção da Saúde relacionadas ao CompHP ainda se mostram exíguas no meio acadêmico. $\mathrm{O}$ fato de o CompHP ter sido desenvolvido no continente Europeu, elaborado e divulgado recentemente, tem evidenciado o ineditismo das produçôes científicas que se ancoram no uso desse referencial. Principalmente quando as concentraçóes de publicaçôes sobre competências para Promoção se concentram na fundamentação de sua importância, ou de seu delineamento e elaboração, sendo escasso publicaçóes de estratégias para implementação na formação profissional.

Para fins de descrição e discussão dos resultados apresentados no quadro 1, evidenciamos duas categorias, Desenvolvimento de Competências do CompHP e Ensino das Competências de PS, respondendo aos achados dos estudos selecionados, os quais foram delineados a partir de dois enfoques: ensino e desenvolvimento de competências para PS (habilidades, conhecimentos, atitudes) durante a formação do enfermeiro.

Em relação ao desenvolvimento das competências definidas no CompHP, os estudos revelam que estas têm sido desenvolvidas durante a formação do enfermeiro no Brasil, ainda que de

Quadro1. Sínteses dos estudos incluídos na revisão integrativa

\begin{tabular}{|c|c|c|c|c|c|c|}
\hline Autoria & Objetivos & Ano & País & $\begin{array}{l}\text { Periódico / área / } \\
\text { Base de Dados }\end{array}$ & $\begin{array}{l}\text { Delineamento/ } \\
\text { Nível de } \\
\text { evidência } \\
\end{array}$ & Principais resultados \\
\hline 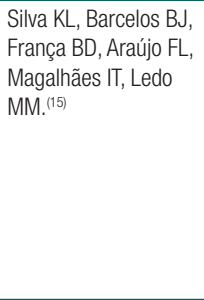 & $\begin{array}{l}\text { Analisar o ensino das } \\
\text { competências para a PS na } \\
\text { formação do enfermeiro, } \\
\text { identificando experimentações } \\
\text { e experiências nesse processo. }\end{array}$ & 2018 & Brasil & \begin{tabular}{|l} 
Interface \\
comunicação, \\
saúde e educação \\
/ Educação e \\
pesquisa em \\
educação de saúde \\
pública e ambiental/ \\
SciELO
\end{tabular} & $\begin{array}{l}\text { Estudo } \\
\text { Qualitativo/ } \\
\text { VI }\end{array}$ & $\begin{array}{l}\text { A partir do estudo das competências essenciais do CompHP, evidenciou- } \\
\text { se que as competências são temporalmente ensinadas no contato com } \\
\text { a realidade, por meio de experimentações e experiências. Os resultados } \\
\text { indicam que o ensino das competências para a PS e ocorre em diferentes } \\
\text { momentos e espaços da formação do enfermeiro, porém a experimentação } \\
\text { da PS é determinada por um local e um tempo específico na formação. A } \\
\text { prática social no campo da formação das competências é ainda marcada pelo } \\
\text { modelo tradicional do ensino e da concepção de PS. O ensino da PS e suas } \\
\text { competências ocorre de modo generalista, não sendo, contudo, especificados } \\
\text { quais domínios devem ser alcançados. }\end{array}$ \\
\hline $\begin{array}{l}\text { Netto, L } \\
\text { Silva, KL. }{ }^{(16)}\end{array}$ & $\begin{array}{l}\text { Analisar a inserção da } \\
\text { prática profissional reflexiva } \\
\text { como estratégia para } \\
\text { o desenvolvimento de } \\
\text { competências para PS na } \\
\text { formação do enfermeiro. } \\
\end{array}$ & 2018 & Brasil & $\begin{array}{l}\text { Revista da Escola de } \\
\text { Enfermagem da USP } \\
\text { - Enfermagem } \\
\text { SciELO, LILACS } \\
\text { CINAHL }\end{array}$ & $\begin{array}{l}\text { Estudo } \\
\text { Qualitativo/ } \\
\text { VI }\end{array}$ & $\begin{array}{l}\text { Os contextos mais favoráveis à prática reflexiva para o desenvolvimento das } \\
\text { competências para a PS são os que comportam a integração ensino-serviço- } \\
\text { comunidade, a interação professor-estudante e o trabalho em equipe. } \\
\text { Os discursos dos participantes revelam imprecisão conceitual a respeito da PS. }\end{array}$ \\
\hline $\begin{array}{l}\text { Dias IKR, Teixeira OF, } \\
\text { Teodoro IP, Maia ER, } \\
\text { Lopes MV, Machado } \\
\text { MS. } .^{(17)}\end{array}$ & $\begin{array}{l}\text { Verificar as perspectivas de } \\
\text { docentes de um curso de } \\
\text { graduação em enfermagem } \\
\text { acerca dos domínios do } \\
\text { CompHP, e como estes estão } \\
\text { presentes em sua prática. }\end{array}$ & 2018 & Brasil & \begin{tabular}{|l|} 
Cogitare \\
Enfermagem- \\
Enfermagem \\
CINAHL, LILACS
\end{tabular} & $\begin{array}{l}\text { Estudo } \\
\text { Qualitativo/ } \\
\text { VI }\end{array}$ & $\begin{array}{l}\text { Os domínios do CompHP puderam ser evidenciados na prática docente. Dentre } \\
\text { os nove domínios estudados, somente quatro se manifestaram nos saberes } \\
\text { profissionais. São eles: favorecimento de mudanças, parceria, liderança, e } \\
\text { diagnóstico. Nenhum docente demonstrou ter propriedade em mais de um } \\
\text { domínio do CompHP }\end{array}$ \\
\hline $\begin{array}{l}\text { Carvalho VL, Oliveira } \\
\text { AL, Alves IK, Silva RL, } \\
\text { Silva CB. }{ }^{(18)}\end{array}$ & $\begin{array}{l}\text { Avaliar o grau de } \\
\text { competências de PS dentre } \\
\text { os formandos dos cursos da } \\
\text { área da saúde. }\end{array}$ & 2017 & Brasil & $\begin{array}{l}\text { Revista enfermagem } \\
\text { UFPE on-line/ } \\
\text { Ciências da saúde- } \\
\text { Enfermagem/ } \\
\text { BDENF }\end{array}$ & $\begin{array}{l}\text { Estudo } \\
\text { Quantitativo/ } \\
\text { VI }\end{array}$ & $\begin{array}{l}0 \text { estudo utilizou um questionário adaptado, composto pelo conjunto dos } 11 \\
\text { domínios de competências do CompHP para avaliar o grau de competências } \\
\text { em promoção da saúde e aponta um desenvolvimento aquém do desejável, no } \\
\text { que diz respeito às competências necessárias para realização das atividades } \\
\text { de PS, durante os cursos de graduação pesquisados. Este fato foi percebido } \\
\text { quando os estudantes afirmaram não reconhecer tais competências. }\end{array}$ \\
\hline $\begin{array}{l}\text { Silva KL, Gonçalves } \\
\text { GA, Santos SB, } \\
\text { Machado MF, } \\
\text { Rebouças CB, Silva } \\
\text { VM, Ximenes LB. }{ }^{(19)}\end{array}$ & $\begin{array}{l}\text { Reconhecer os domínios } \\
\text { de competências de PS } \\
\text { no processo formativo de } \\
\text { adolescentes realizado por } \\
\text { acadêmicos de enfermagem. }\end{array}$ & 2018 & Brasil & $\begin{array}{l}\text { Revista Brasileira } \\
\text { de Enfermagem / } \\
\text { Ciências da saúde - } \\
\text { Enfermagem } \\
\text { SciELO, PUBMED, } \\
\text { LILACS, BDENF, } \\
\text { WOS, CINAHL } \\
\end{array}$ & $\begin{array}{l}\text { Estudo } \\
\text { Qualitativo/ } \\
\text { VI }\end{array}$ & $\begin{array}{l}\text { Evidenciaram-se quatro domínios das competências previstas no modelo } \\
\text { CompHP: Mudança Ativa; Mediar através de Parceria; Comunicação; e } \\
\text { Liderança }\end{array}$ \\
\hline $\begin{array}{l}\text { Netto L, Silva KL, Rua } \\
\text { MS. }{ }^{(20)}\end{array}$ & $\begin{array}{l}\text { Analisar o desenvolvimento } \\
\text { de competências para PS, na } \\
\text { perspectiva dos egressos de } \\
\text { um curso de enfermagem, e } \\
\text { sua relação com o modelo de } \\
\text { atenção. }\end{array}$ & 2016 & Brasil & $\begin{array}{l}\text { Texto Contexto } \\
\text { Enfermagem / } \\
\text { Ciências da saúde - } \\
\text { Enfermagem/ } \\
\text { SciELO }\end{array}$ & $\begin{array}{l}\text { Estudo } \\
\text { Qualitativo } \\
\text { VI }\end{array}$ & $\begin{array}{l}\text { As competências para PS, definidas nos consensos Galwey e CompHP, foram } \\
\text { trabalhadas ao longo do processo de formação. } \\
\text { Os discursos produzidos demonstram que o desenvolvimento de competências } \\
\text { para PS pode favorecer a mudança do modelo assistencial, apesar dos desafios } \\
\text { neste processo. } \\
\text { As competências de advocacia em saúde, possibilidade de mudança, liderança } \\
\text { e parcerias estão presentes nos discursos. }\end{array}$ \\
\hline
\end{tabular}


forma irregular e aquém do desejável. ${ }^{(18)}$ Dos 11 domínios do projeto, apenas três deles tiveram regularidade nos estudos que buscaram reconhecer o desenvolvimento dos domínios de competências do CompHP na formação do enfermeiro no cenário brasileiro. ${ }^{(17,19,20)}$ São eles: Possibilidade de mudança, Parceria e Liderança, sendo comuns nos discursos de estudantes e docentes, e indicativos de mudança no modelo de atenção à saúde, ainda que tenhamos muitos desafios nesse processo. ${ }^{(17,19,20)}$

No que diz respeito às competências do domínio Possibilidade de Mudança, os estudos evidenciam que estas estão sendo desenvolvidas durante a formação do enfermeiro, mesmo que não estejam incorporadas aos currículos e projetos pedagógicos de forma explícita. ${ }^{(18,19)}$

Os estudos incluídos nesta revisão, demonstram por meio dos discursos dos estudantes e dos saberes e práticas dos docentes, o reconhecimento e a importância do trabalho colaborativo para o impacto positivo das açóes de PS na vida da população. ${ }^{(15,16,20)}$

A análise dos estudos incluídos nesta revisão revelam um modelo de ensino em transição, com avanços nos processos de formação e educação profissional, por meio de experiências inovadoras, propiciando a imersão na realidade profissional nos períodos iniciais de formação, a interdisciplinaridade e uma prática profissional crítica, reflexiva e colaborativa entre serviço-comunidade-professor-aluno. ${ }^{(16)}$

Apesar dos avanços na área, o ensino da PS é marcado pela racionalidade técnica, a fim de consolidar o conteúdo teórico, em que a prática é regulada pelos professores, propiciando uma experimentação de um SUS ideal e não real, oferecendo, dessa forma, poucas possibilidades de experiências. ${ }^{(15)}$ Ademais, no contexto brasileiro, evidenciou-se que docentes de graduação em enfermagem não demonstram ter propriedade quanto aos domínios do CompHP, apesar de estes serem evidenciados na prática docente. ${ }^{(17)}$

\section{Discussão}

Os resultados desta revisão permitiram evidenciar que há uma produção científica que discute a formação acadêmica do enfermeiro com aporte do
Projeto CompHP na realidade brasileira, demonstrando assim um interesse da profissão em contribuir para um olhar integrado em saúde e superação do paradigma biologicista ainda presente nas práticas de saúde. É importante destacar que apesar de o enfoque do estudo ter sido a realidade nacional, não foram evidenciados, estudos internacionais que discutam a formação acadêmica do enfermeiro com aporte do Projeto CompHP. O que se encontrou, no entanto, foram estudos de implementação do CompHP no continente Europeu, com menção ao uso do projeto na educação em saúde para PS e em cursos acadêmicos profissionais, por exemplo medicina e psicologia, mas não em cursos de Enfermagem. ${ }^{(21,22)}$

\section{Desenvolvimento de competências do CompHP na formação do enfermeiro}

De acordo com o CompHP, o domínio Possibilidade de mudança "visa possibilitar que indivíduos, grupos, comunidades ou organizaçóes construam capacidade para ação em PS, e assim, melhorar a saúde e reduzir as iniquidades em saúde". ${ }^{(8)}$ A saúde é um recurso para a vida e, para que se atinja seu potencial, é imperativa a capacitação por meio de educação em saúde e divulgação de informaçóes com linguagem apropriada. Além disso, o Estado deve oferecer recursos e oportunidades que permitam uma escolha saudável. ${ }^{(23)} \mathrm{O}$ desenvolvimento de competências referente ao domínio Parceria envolve o trabalho colaborativo com áreas de conhecimento, setores e parceiros distintos, a fim de aumentar o impacto e a sustentabilidade das açóes de PS; uma vez que a concepção ampliada de saúde não é assegurada somente pelo setor saúde, mas requer uma ação coordenada entre setores governamentais e não-governamentais. ${ }^{(8,23)}$ Os estágios, projetos de pesquisa e extensão e o envolvimento de espaços ainda pouco explorados, como igrejas, associaçóes comunitárias, e escolas, podem propiciar maior engajamento civil.

Nota-se que a liderança é reconhecida como uma competência geral do enfermeiro, sendo fundamental para seu exercício de trabalho. Uma vez que este profissional atua liderando equipes de enfermagem e agentes comunitários de saúde, bem como, por sua essência de trabalho ser o cuidado é 
o elo de comunicação entre equipe multidisplinar, pacientes e instituiçốes. ${ }^{(24)} \mathrm{A}$ liderança pode ser desenvolvida e aperfeiçoada na graduação, para isso, são imprescindíveis metodologias que estimulem a aquisição de habilidades referentes à resolução de conflitos; o gerenciamento de recursos; o trabalho em equipe com diversos setores e parceiros; a negociação; e tomada de decisão, a fim de formar redes de pessoas-chave motivadas a contribuir numa visão compartilhada em açôes de PS. ${ }^{(8,17)}$

É importante ressaltar que um dos estudos incluídos nesta revisão revela um conceito reduzido sobre Promoção da Saúde, com menção majoritária à prevenção de doenças. ${ }^{(16)}$ Esse fato pode dificultar o processo de desenvolvimento das Competências de PS na formação do enfermeiro, uma vez que esse domínio é considerado um pilar e permeia todas as competências e demais domínios delineados no projeto. ${ }^{(8)}$ É preciso superar a perspectiva biologicista e preventivista que desvincula a dimensão política vigente na área. ${ }^{(25)}$

\section{Ensino das competências de promoção da saúde}

As Diretrizes Curriculares Nacionais (DCN's) apontam para uma necessidade de integração dos conteúdos curriculares, pois favorece o processo de ensino-aprendizagem e atuação em equipe multidisciplinar, porém, há dificuldade e resistência em operacionalizar essa integração, ${ }^{(15,24,26)}$ uma vez que o ensino das competências de PS tem sido feito de forma geral e reduzido, principalmente, a disciplinas de saúde coletiva. ${ }^{(15)}$

A fragilidade nos saberes docentes referente aos domínios do CompHP, identificados neste estudo, enfraquece a formação dos profissionais de enfermagem; visto que, alguns domínios como a Advocacia em saúde, são fundamentais às reivindicações a favor dos usuários, sendo reconhecidos como norteadores da PS desde a concepção da Carta de Ottawa. ${ }^{(17,23)}$

É preciso formar profissionais com competência em pleitear, junto aos diversos setores da sociedade, pelo desenvolvimento de políticas públicas, com capacidade em sensibilizar a opinião da população e, aliado a pessoas-chave, crie engajamento a fim influenciá-las a desenvolver e manter açóes de PS, que impactem positivamente os diversos condicionantes de saúde. ${ }^{(8,23)}$
No Brasil, entende-se a necessidade da formação de trabalhadores para a saúde capazes de assegurar os princípios e diretrizes do Sistema Único de Saúde (SUS) e da PNPS. ${ }^{(3,4,27)}$ Nos últimos anos tem aumentado a busca por capacitação nesse campo, e algumas instituições renomadas têm criado cursos de especialização lato e stricto sensu na área. ${ }^{(28)}$ A PS é uma competência geral na formação do enfermeiro, em que o conhecimento e as habilidades na área refletem-se na conduta e exercício profissional. ${ }^{(24)}$

Nesse sentido, inúmeros esforços têm sido feitos ao longo dos últimos anos, como o estímulo à criação de novas propostas pedagógicas, que propiciem uma formação crítica-reflexiva e desenvolva competências, capazes de romper a dissociação teoria-prática ainda existente e mudar o modelo de saúde assistencial. ${ }^{(26)}$ Dentre elas, algumas têm sido proveitosas ao desenvolvimento de competências de PS, como o uso de portfólios reflexivos, visitas domiciliares, simulação realística, mapas conceituais e estudos de caso. ${ }^{(29,30)}$

Contudo, há dificuldades em relação ao uso de metodologias de ensino diferenciadas, que propiciem o desenvolvimento de competências éticas, políticas e sociais, devido à falta de formação de professores para o uso de metodologias ativas. ${ }^{(26,29)}$ Além disso, as DCNs não deixam explícito o conteúdo para a elaboração de Competências para PS, apenas direcionam o perfil profissional, sendo que as instituiçôes formadoras têm a responsabilidade de estimular essas competências em seus alunos. ${ }^{(24,26)}$

Um estudo de revisão escopo corrobora os resultados de nosso estudo, demonstrando a escassez de pesquisas de avaliação sobre impacto na prática, educação e treinamento, sendo raros os estudos sobre implementação e uso de competências de promoção da saúde relacionados ao CompHP. ${ }^{(21)}$ De maneira geral, as produçóes científicas sobre Competências para PS se concentram na descrição de seus processos de construção e nos resultados positivos esperados de suas implementações. ${ }^{(21,22)}$

Dessa forma, a fim de superar esses aspectos, acredita-se que a realidade brasileira possa se beneficiar com o CompHP, mesmo que este tenha sido desenvolvido em outra realidade sociocultural. Pesquisas nacionais têm sido proveitosas e mostram que, com 
adequaçóes e ajustes do referencial à realidade brasileira, é possível atender aos objetivos das DCN's e políticas de saúde do país. ${ }^{(31)}$ Dessa forma, acreditamos que os currículos dos cursos de Enfermagem podem ser apoiados pelo CompHP, a fim de nortear a formação de futuros enfermeiros e assegurar o fortalecimento da Enfermagem na Área de PS.

O fato de encontrarmos publicações com nível de evidência VI destaca a necessidade de estudos de outra natureza, com representaçóes que reflitam adequadamente o perfil de Instituiçóes de Ensino Superior de Enfermagem no país, a fim de propiciar uma melhor compreensão e avaliação dos impactos das competências para promoção da saúde do CompHP na prática, educação e treinamento.

\section{Conclusão}

Há evidências na literatura que os domínios de competências de PS relacionados ao CompHP têm sido desenvolvidas durante a formação do enfermeiro na realidade brasileira, por meio de experiências práticas e estratégias curriculares inovadoras. Contudo, os dados ainda são exploratórios e não há uma regularidade no desenvolvimento dessas competências durante o processo de formação do enfermeiro. Internacionalmente, ao longo dos anos tem sido debatido e delineado competências de PS, com vistas para uma realidade multicultural, mas ainda há escassez de estudos que mostrem como essas competências podem ser desenvolvidas e aplicadas na prática profissional. Assim, esse estudo apresenta experiências do desenvolvimento de competências para PS e evidências de implementação e de estratégias de ensino que contribuem para formaçáo profissional a partir da perspectiva da CompHP. Como no cenário brasileiro, ainda temos um referencial de competências para PS, sendo possível associar competências do CompHP durante a formação de estudantes de enfermagem, acredita-se que as competências delineadas no projeto CompHP podem ser perfeitamente adaptadas para guiar a formação de profissionais de saúde, podendo ser incorporados aos curriculos e Projeto pedagógigo por meio de um ensino que busque atingir os conhecimen- tos e habilidades mínimos em PS, já identificados e propostos pelo CompHP, com a perspectiva de formar profissionais capazes de prestar um cuidado em saúde mais eficiente e qualificado, com melhorias em todos os níveis de atenção. Espera-se que esse estudo de revisão amplie a discussão das DCNs em enfermagem que estão em constante para construção e aprimoramento para que possamos assegurar padróes profissionais, utilizando mecanismos que assegurem a qualidade e o fortalecimento da Enfermagem na área de Promoção da Saúde. Ao mesmo tempo, ele pode ser utilizado para subsidiar discussões nas equipes de saúde sobre as competências necessárias para o cuidado, bem como podem ser idealizadas pesquisas empíricas sobre a implementação das competências exploradas em tela.

\section{Referências}

1. Malta DC, Morais Neto OL, Silva MM, Rocha D, Castro AM, Reis AA, Akerman M. [National Health Promotion Policy (PNPS): chapters of a journey still under construction]. Ciênc Saúde Coletiva. 2016; 21(6):1683-94. Portuguese

2. Feio A, Oliveira CC. Confluências e divergências conceituais em educação em saúde. Saude Soc. 2015;24(2):703-15.

3. Brasil. Ministério da Saúde. Política Nacional de Promoção da Saúde. Portaria $n^{0} 687$ de 30 de março de 2006]. Brasília (DF): [Internet]; 2016. [citado 2018 Out 8]. Disponivel em: :http://189.28.128.100/ dab/docs/legislacao/portaria687_30_03_06.pdf

4. Pinheiro DG, Scabar TG, Maeda ST, Fracolli LA, Pelicioni MC, Chiesa AM. Competências em promoção da saúde: desafios da formação. Saude Soc. 2015;24(1):180-8.

5. Barry MM, Battel-Kirk B, Dempsey C. The CompHP core competencies framework for health promotion in Europe. Health Educ Behav. 2012;39(6):648-62.

6. Barry MM, Battel-Kirk B, Davison H, Dempsey C, Parish R. Schipperen, et al. The CompHP Project Handbooks. International Union for Health Promotion and Education. Paris: IUHPE; 2012.

7. Battel-Kirk B, Barry MM, van der Zanden G, Contu P, Gallardo C, Martinez A, et al. Operationalising and piloting the IUHPE European accreditation system for health promotion. Glob Health Promot Educ. 2015;22(3):25-34.

8. Dempsey C, Battel-Kirk B, Barry MM. comp. The CompHP Core Competencies Framework for Health Promotion Handbook. ComHP; 2011.

9. Silva KL, Araujo FL, Santos FB, Andrade AM, Basilio NC, Sena RR. 0 que vem se falando por aí em competências no ensino da promoção da saúde na formação do enfermeiro? ABCS Health Sci. 2015;40(3):286-93.

10. Silva PF, Baptista TW. Os sentidos e disputas na construção da Política Nacional de Promoção da Saúde. Physis Rev Saúde Coletiva. 2014;24(2):441-65. 
11. Mendes KD, Silveira RC, Galvão CM. Revisão integrativa: método de pesquisa para a incorporação de evidências na saúde e na enfermagem. Texto Contexto Enferm. 2008;17(4):758-64.

12. Biruel E, Pinto R. Bibliotecário um profissional a serviço da Pesquisa. XXIV Congresso Brasileiro de Biblioteconomia, Documentação e Ciência da Informação. Maceió. Anais. 2011[citado 2019 Jun 4] Disponível em from: https://www.academia.edu/9594560/Bibliotec\%C3\%A1rio_ um_profissional_a_servi\%C3\%A70_da_pesquisa

13. Shamseer L, Moher D, Clarke M, Ghersi D, Liberati A, Petticrew M, et al.; PRISMA-P Group. Preferred reporting items for systematic review and meta-analysis protocols (PRISMA-P) 2015: elaboration and explanation. BMJ. 2015;350 jan02 1:g7647.

14. Melnyk BM, Fineout-Overholt E. Evidence-based practice in nursing \& healthcare: A guide to Best practice. Lippincott Williams \& Wilkins; 2011.

15. Silva KL, Barcelos BJ, França BD, Araújo FL, Magalhães IT, Ledo MM. [Between experiments and experiences: challenges for teaching competencies for health promotion in Nursing Education]. Interface (Maynooth). 2018;22(67):1209-20. Portuguese.

16. Netto L, Silva KL. [Reflective practice and the development to competencies for health promotion in nurses' training]. Rev Esc Enferm USP. 2018;52(3):1-9. Portuguese.

17. Dias IK, Teixeira OF, Teodoro IP, Maia ER, Lopes MV, Machado MS. [Nursing educators' perceptions of the domains of the core Competencies framework for health promotion]. Cogitare Enferm . 2018 ]; 23(2):1-9. Portuguese

18. Carvalho VL, Oliveira AL, Alves IK, Silva RL, Silva CB. [Health promotion competencies of undergraduate health professionals] Rev Enferm UFPE. 2017;11(8):3269-78. Portuguese.

19. Silva KL, Gonçalves GA, Santos SB, Machado MF, Rebouças CB, Silva VM, et al. Características dos enfermeiros de uma unidade tecnológica: implicações para o cuidado de enfermagem. Rev Bras Enferm. 2018;71(1):98-105.
20. Netto L, Silva KL, Rua MS. Competency building for health promotion and change in the care model. Texto Contexto Enferm. 2016;25(2):2-7.

21. Battel-Kirk B, Barry MM. Has the Development of Health Promotion Competencies Made a Difference? A Scoping Review of the Literature. Health Educ Behav. 2019;46(5):824-42.

22. Battel-Kirk B, Barry MM. Implementation of Health Promotion Competencies in Ireland and Italy-A Case Study. Int J Environ Res Public Health. 2019;16(24):E4992.

23. World Health Organization (WHO). The Ottawa charter for health promotion. Ottawa: WHO; 2000.

24. Brasil. Ministério da Educação. Diretrizes curriculares nacionais do curso de graduação em enfermagem. Brasília (DF): Ministério da Educação; 2001.

25. Santos SK, Da Ros MA. Ressignificando Promoção de Saúde em Grupos para Profissionais da Saúde. Rev Bras Educ Med. 2016;40(2):189-96.

26. Mattia BJ, Kleba ME, Prado ML. Nursing training and professional practice: an integrative review of literature. Rev Bras Enferm. 2018;71(4):2039-49.

27. Brasil. Ministério da Saúde e Educação. Programa Nacional de Reorientação da Formação Profissional em Saúde. Objetivos, implementação e desenvolvimento potencial. Brasília (DF): Ministério da Saúde e Educação; 2007.

28. Tavares MF, Rocha RM, Bittar CM, Petersen CB, Andrade M. Health promotion in professional education: challenges in Health and the need to achieve in other sectors. Cien Saude Colet. 2016;21(6):1799-808.

29. Netto L, Silva KL, Rua MS, Sena RR. The process of teaching competencies for health promotion] .Rev Enferm Cent O Min. 2018;8(1): e2611. Portuguese.

30. Brown CL. Linking public health nursing competencies and servicelearning in a global setting. Public Health Nurs. 2017;34(5):485-92.

31. Moreira MRC, Machado MFAS. Matrix of essential competencies in health promotion: a proposal for the Brazilian context. Health Promot Int. 2019 0ct 3:daz096. 